\title{
Efficacy and safety of leuprorelin acetate 6-month depot, TAP- 144-SR (6M), in combination with tamoxifen in postoperative, premenopausal patients with hormone receptor-positive breast cancer: a phase III, randomized, open-label, parallel-group comparative study
}

\author{
Junichi Kurebayashi $^{1} \cdot$ Tatsuya $^{\text {Toyama }^{2}} \cdot$ Shuuji Sumino $^{3} \cdot$ Eri Miyajima $^{3}$ • \\ Tsukasa Fujimoto ${ }^{3}$
}

Received: 24 December 2015/ Accepted: 15 March 2016/Published online: 26 March 2016

(c) The Author(s) 2016. This article is published with open access at Springerlink.com

\begin{abstract}
Background Leuprorelin acetate, a luteinizing hormonereleasing hormone agonist, is used worldwide in premenopausal women with hormone receptor-positive breast cancer. This study was conducted to assess the non-inferiority of the 6-month depot formulation, TAP-144-SR (6M) $22.5 \mathrm{mg}$ to the 3-month depot formulation, TAP-144SR (3M) $11.25 \mathrm{mg}$ in postoperative, premenopausal patients with hormone receptor-positive breast cancer.

Methods This was a 96-week phase III, randomized, open-label, parallel-group comparative study. All patients concomitantly received oral tamoxifen ( $20 \mathrm{mg}$ daily). The primary endpoint was the suppression rate of serum estradiol $\left(\mathrm{E}_{2}\right)$ to the menopausal level $(\leq 30 \mathrm{pg} / \mathrm{mL})$ from Week 4 through Week 48.

Results In total, 167 patients were randomized to receive TAP-144-SR $(6 \mathrm{M}) \quad(n=83)$ or TAP-144-SR $(3 \mathrm{M})$ $(n=84)$ and the $\mathrm{E}_{2}$ suppression rate was 97.6 and $96.4 \%$, respectively. The estimated between-group difference was $1.2 \%$ (95\% confidence interval -5.2 to 7.8 ). The noninferiority of TAP-144-SR (6M) to TAP-144-SR (3M) for $\mathrm{E}_{2}$ suppression was confirmed. As for safety, common adverse events were hot flush and injection site reactions including induration, pain, and erythema in both treatment groups, which were of $\leq$ Grade 2 in severity and not
\end{abstract}

Junichi Kurebayashi

kure@med.kawasaki-m.ac.jp

1 Department of Breast and Thyroid Surgery, Kawasaki Medical School, 577 Matsushima, Kurashiki,

Okayama 701-0192, Japan

2 Department of Breast and Endocrine Surgery, Nagoya City University Hospital, Nagoya, Aichi, Japan

3 Takeda Pharmaceutical Company Limited, Osaka, Japan serious. No significant between-group differences in safety profiles and tolerability were observed.

Conclusions TAP-144-SR (6M) was not inferior to TAP144-SR (3M) for its suppressive effect on serum $E_{2}$. TAP144-SR (6M) was also as well tolerated as TAP-144-SR (3M).

Keywords Leuprorelin acetate 6-month depot . Luteinizing hormone-releasing hormone agonist . Premenopausal hormone receptor-positive breast cancer . Adjuvant endocrine therapy · Ovarian function suppression

\section{Introduction}

For the treatment of premenopausal women with hormone receptor-positive breast cancer, it is highly important to suppress estrogen production through ovarian function suppression (OFS). OFS therapy with a luteinizing hormone-releasing hormone (LH-RH) agonist, in combination with adjuvant tamoxifen or chemotherapy is widely used for postoperative premenopausal endocrine-responsive breast cancer patients [1-7]. However, the optimal treatment duration of postoperative adjuvant endocrine therapy with an LH-RH agonist alone or in combination with tamoxifen is still controversial [8-10]. Although premenopausal hormone receptor-positive breast cancer patients have a relatively good prognosis, the risk of recurrence remains at 5 years or longer after surgery, suggesting the importance of long-term endocrine therapy for 5 years or longer for the treatment of patients at high risk for recurrence [11].

Leuprorelin acetate (leuprorelin), an LH-RH agonist is commonly used for the treatment of patients with hormoneresponsive prostate cancer and premenopausal breast 
cancer worldwide. It is available in 1- and 3-month depot formulations for both cancers, and a 6-month depot formulation for prostate cancer.

A 6-month depot formulation, TAP-144-SR (6M), was initially developed for prostate cancer in Japan and a phase II study was conducted in treatment-naïve prostate cancer patients. The results showed that the optimal clinical dosage of TAP-144-SR (6M) in Japan is $22.5 \mathrm{mg}$ [12].

In parallel with the phase III study in prostate cancer, this phase III study was also conducted in Japan for the first time to assess the non-inferiority of TAP-144-SR (6M) $22.5 \mathrm{mg}$ to TAP-144-SR (3M) $11.25 \mathrm{mg}$ regarding its suppressive effect on serum estradiol $\left(\mathrm{E}_{2}\right)$, and to evaluate its efficacy and safety in postoperative, premenopausal patients with hormone receptor-positive breast cancer.

\section{Patients and methods}

\section{Study design}

A phase III, randomized, open-label, parallel-group comparative study of TAP-144-SR (6M) to TAP-144-SR (3M) was conducted to evaluate the efficacy, safety, and pharmacokinetics of the 2 formulations and hormone levels in postoperative, premenopausal patients with endocrine-responsive breast cancer. Following a 4-week screening period, eligible patients were randomly assigned at a $1: 1$ ratio to receive injection of either TAP-144-SR (6M) $22.5 \mathrm{mg}(6 \mathrm{M}$ group) or TAP-144-SR (3M) $11.25 \mathrm{mg}$ (3M group) for 96 weeks using dynamic allocation with the number of positive axillary lymph nodes $(0,1-3, \geq 4)$, tumor diameter $(\leq 2.0,>2.0 \mathrm{~cm})$, estrogen receptor $(\mathrm{ER}) /$ progesterone receptor $(\mathrm{PgR})$ status $(\mathrm{ER}+/ \mathrm{PgR}+, \mathrm{ER}+/ \mathrm{PgR}-, \mathrm{ER}-/$ $\mathrm{PgR}+$ ), age (at the time of consent; $\leq 39,40-44, \geq 45$ years), pre- and post-operative chemotherapy (presence, absence), and study site as factors. All patients in both groups concomitantly received oral tamoxifen citrate (20 mg daily) throughout the 96-week study period.

This study was conducted in accordance with the International Conference on Harmonisation of Good Clinical Practice Guidelines, the principles of the Declaration of Helsinki, and all applicable laws and regulations, at 20 medical centers in Japan between April 2012 and December 2014. The protocol was reviewed and approved by the Institutional Review Boards of all study participating sites. All patients provided written informed consent before enrollment. The clinical trial registration number is NCT01546649.

\section{Patients}

Japanese premenopausal patients with histologically confirmed primary breast cancer who met the following criteria were eligible: age $\geq 20$ years; both or either ER+ or $\mathrm{PgR}+$, and human epidermal growth factor receptor type 2 (HER-2)-negative primary tumor; T1-T3, any N, and M0 according to the TNM classification; any type of surgical procedure (in case of breast-conserving surgery, postoperative radiation to the breast was required); any type of preoperative and/or postoperative adjuvant chemotherapy prior to enrollment; history of regular menstruation or follicle-stimulating hormone (FSH) of $<40 \mathrm{mIU} / \mathrm{mL}$ and $\mathrm{E}_{2}$ of $\geq 10 \mathrm{pg} / \mathrm{mL}$ within 12 weeks prior to enrollment and not having chemical menopause (FSH of $\geq 40 \mathrm{mIU} / \mathrm{mL}$ and $\mathrm{E}_{2}$ of $<10 \mathrm{pg} / \mathrm{mL}$ ) within 12 weeks after completion of the postoperative chemotherapy; capable of receiving the study drug and tamoxifen within 12 weeks after surgery or after postoperative chemotherapy completion prior to enrollment; Eastern Cooperative Oncology Group performance status of Grade 0 or 1.

Exclusion criteria included the following: endocrine therapy prior to surgery or postoperative endocrine therapy before enrollment; bilateral oophorectomy or ovarian irradiation; inflammatory breast cancer or bilateral breast cancer; non-invasive ductal carcinoma, multiple cancers or a history of cancer in other organs; QTcF interval exceeding $460 \mathrm{~ms}$ on the 12-lead electrocardiogram (ECG) at screening.

\section{Primary and secondary endpoints}

The primary endpoint was the suppression rate of serum $\mathrm{E}_{2}$ to the menopausal level $(\leq 30 \mathrm{pg} / \mathrm{mL})$, which is the best index of the medicinal effect of leuprorelin, from Week 4 through Week 48. The secondary endpoints included: serum $\mathrm{E}_{2}, \mathrm{LH}$, and FSH concentrations; disease-free survival [DFS; defined as the time from random assignment to disease event (recurrence, second primary cancer, or death)] and distant DFS [DDFS; defined as the time from random assignment to disease event (distant recurrence, second primary cancer, or death)] throughout the study period as measures of the longterm efficacy. All the serum hormone concentrations were measured at a central laboratory (SRL Medisearch Inc., Tokyo, Japan). Electrochemiluminescence immunoassay for $\mathrm{E}_{2}$ (ECLusys ${ }^{\circledR}$ E2III, Roche Diagnostics K.K., Tokyo, Japan), chemiluminescence immunoassay for LH (ARCHITECT $^{\circledR}$. LH, Abbott Japan, Chiba, Japan) and chemiluminescence immunoassay for FSH (ARCHITECT ${ }^{\circledR}$ - FSH, Abbott Japan, Chiba, Japan) were used to measure each serum hormone concentration.

Pharmacokinetic analysis was performed by measuring the serum concentrations of unchanged TAP-144 using LC/ MS/MS from the start of the study drug administration through Week 48.

Safety data were obtained from the findings of clinical signs/symptoms, body weight, vital signs, laboratory test 
results, 12-lead ECG, and bone mineral density (BMD) measured by dual-energy X-ray absorptiometry throughout the study period. Adverse events (AEs) were recorded and graded according to the National Cancer Institute Common Terminology Criteria for Adverse Events version 4.0.

\section{Statistical analysis}

The required sample size was estimated as 74 subjects in each treatment group, a total of 148 subjects, based on which the conditions were set as a non-inferiority margin of $10 \%$, with a two-sided alpha level of 0.05 , ensuring $80 \%$ power. Taking a possible drop-out rate of $10 \%$ into consideration, 82 patients in each treatment group, thus a total of 164 patients were required.

The Full Analysis Set (FAS) was defined as all patients who were randomized and received at least 1 dose of the study drug, and the Hormone Analysis Set (HAS) was defined as the patients who had no major protocol deviations, and in whom the primary endpoints were evaluable. The FAS was used for the primary and secondary endpoints and the HAS was used to examine the robustness of the results of the primary analysis. The treatment difference [TAP-144-SR (6M) - TAP-144-SR (3M)] and the two-sided $95 \%$ confidence interval (CI) were calculated by a method based on the Wilson score method [13]. If the lower bound of the two-sided $95 \%$ CI was greater than the prespecified non-inferiority margin of $-10 \%$, clinical non-inferiority of TAP-144-SR (6M) to TAP-144SR (3M) would be declared. Summary statistics were obtained for the secondary efficacy endpoints. Serum $E_{2}$ concentrations lower than or equal to the limit of quantification $(10 \mathrm{pg} / \mathrm{mL})$ were considered to be $0 \mathrm{pg} / \mathrm{mL}$. DFS and DDFS were estimated by the Kaplan-Meier method.

For the safety analysis, AEs and their severity were analyzed by treatment group. AEs were summarized in the Safety Data Analysis Set (SAS) defined as all patients who were randomized and received at least 1 dose of the study drug and were coded by the System Organ Class Preferred Terms based on the Medical Dictionary for Regulatory Activities (MedDRA) terminology, version 16.1.

As for pharmacokinetic analysis, summary statistics were calculated for serum unchanged-TAP-144 concentrations through Week 48 in the Pharmacokinetics Analysis Set (PAS; defined as the population of patients in the FAS in whom serum unchanged-TAP-144 concentrations were appropriately measured), and for pharmacokinetic parameters in patients in whom serum unchanged-TAP-144 concentrations were measured at 3 and $6 \mathrm{~h}$ after the study drug administration in the PAS.

\section{Results}

\section{Patient demographics}

Figure 1 shows the patient disposition. Of the 180 patients who provided written informed consent, a total of 167 patients were randomized, 83 patients received TAP-144SR (6M) and 84 patients received TAP-144-SR (3M). Overall, 150 patients (75 patients in each treatment group) completed the 96-week study treatment, and the majority of patients $(92.8$ and $91.7 \%$ in the $6 \mathrm{M}$ and $3 \mathrm{M}$ groups, respectively) received the maximum doses (4 doses and 8 doses in the $6 \mathrm{M}$ and $3 \mathrm{M}$ groups, respectively).

The baseline demographic and disease characteristics of patients are summarized in Table 1. No major differences were observed in the baseline characteristics between the treatment groups.

\section{Efficacy}

\section{$E_{2}$ suppression rate}

For the primary endpoint, the suppression rate of serum $E_{2}$ to the menopausal level $(\leq 30 \mathrm{pg} / \mathrm{mL})$ from Week 4 through Week 48 in the FAS was $97.6 \%$ (95\% CI 91.6-99.7) in the 6M group and $96.4 \%(95 \%$ CI 89.9-99.3) in the 3M group (Table 2). The estimated between-group difference in the suppression rate was $1.2 \%$ (95\% CI -5.2 to 7.8). Since the lower CI was more than the pre-determined non-inferiority margin of $-10 \%$, the non-inferiority of TAP-144-SR (6M) to TAP-144-SR $(3 \mathrm{M})$ was confirmed for the suppressive effect on serum $\mathrm{E}_{2}$. Five patients ( 2 and 3 patients in the $6 \mathrm{M}$ and $3 \mathrm{M}$ groups, respectively) had a serum $E_{2}$ concentration exceeding $30 \mathrm{pg} / \mathrm{mL}$ during the period from the start of study drug administration to Week 48 , which was measured at only 1 assessment time point in each patient. For the sensitivity analysis, the same analysis as for the primary analysis was utilized in the HAS as the secondary analysis. Similar results were obtained in the HAS [between-group difference in the suppression rate, $2.4 \%$ (95\% CI -3.8 to 9.2)]. Therefore, the non-inferiority of TAP-144-SR (6M) to TAP-144-SR (3M) was confirmed in both analysis sets.

\section{Changes in the hormone levels and menstrual status}

The median serum $E_{2}$ concentrations significantly declined to the value of $0 \mathrm{pg} / \mathrm{mL}$, below the menopausal level of $\leq 30 \mathrm{pg} / \mathrm{mL}$ from Week 4 through Week 48 (Fig. 2), and remained at the suppressed level until Week 96 in both treatment groups. 
Fig. 1 Patient disposition. $n$ number of patients evaluated, $A E$ adverse event

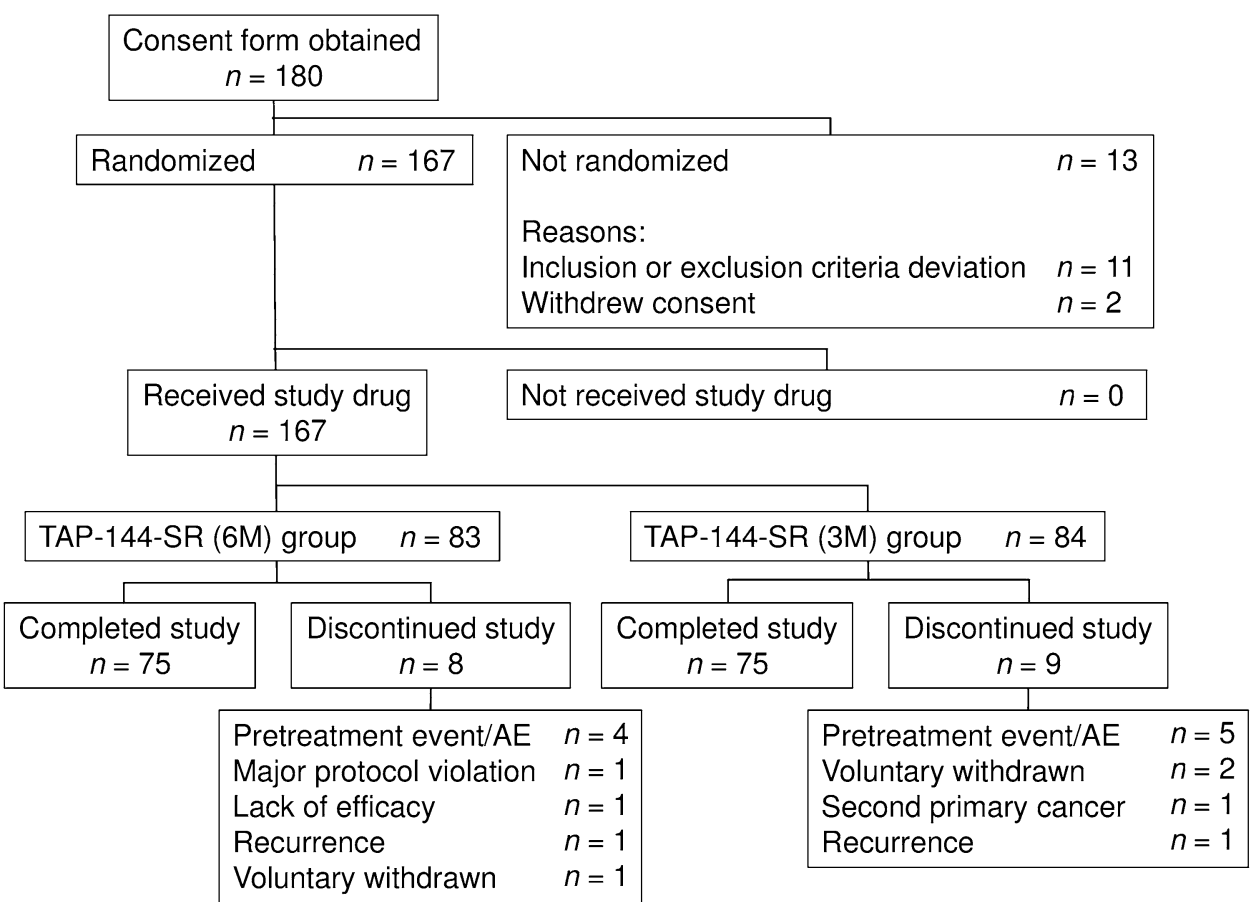

Similarly, the median serum LH and FSH concentrations were suppressed to the levels of $\leq 1$ and $\leq 2.5 \mathrm{mIU} / \mathrm{mL}$, respectively from Week 4 , and remained at the low levels through Week 96 in both treatment groups. There were no significant differences in the changes in these hormone levels between the treatment groups.

Throughout the study period, all patients achieved amenorrhea from Week 8, except 1 patient in the 6M group who had menses at Week 8.

\section{$D F S$ and DDFS}

Throughout the 96-week study period, there were 4 disease events ( 2 each in the $6 \mathrm{M}$ and $3 \mathrm{M}$ groups, respectively): 3 recurrences ( 2 and 1 ), and $1 \mathrm{~s}$ primary cancer in the $3 \mathrm{M}$ group. One recurrence in the 6M group was bone metastasis. The DFS rate at Week 96 in the FAS was $97.3 \%(95 \% \mathrm{CI}$ 93.6-100.0) and 97.5\% (95\% CI 94.1-100.0) in the 6M and $3 \mathrm{M}$ groups, respectively, with no significant between-group differences (estimated difference, $-0.2 \%$ [95 \% CI -5.2 to 4.9]). The DDFS rate at Week 96 in the FAS was $98.5 \%$ (95\% CI 95.7-100.0) and 98.8\% (95\% CI 96.4-100.0) in the $6 \mathrm{M}$ and $3 \mathrm{M}$ groups, respectively. There were no significant differences between the treatment groups (estimated difference, $-0.3 \%$ [95 \% CI -4.0 to 3.4]).

\section{Pharmacokinetics}

Serum TAP-144 concentrations rapidly increased immediately after the administration of TAP-144-SR (6M), and then rapidly decreased through Day 8 (Fig. 3). Thereafter, they increased again from Week 2 through Week 3, and gradually declined through Week 24, showing a doublepeak of TAP-144. The profile of serum TAP-144 concentrations after the initial administration was similar to that after the second administration. In contrast, serum TAP144 concentration rapidly increased $1 \mathrm{~h}$ after the administration of TAP-144-SR (3M), and then gradually declined during the period from 3 to $12 \mathrm{~h}$. The maximum drug concentration $\left(C_{\max }\right)$ in the $6 \mathrm{M}$ group was approximately one-fifth of that in the $3 \mathrm{M}$ group, and the area under the blood concentration-time curve in the $6 \mathrm{M}$ group was approximately 1.8 times that in the $3 \mathrm{M}$ group (data not shown). No obvious accumulation was observed either with TAP-144-SR (6M) or TAP-144-SR (3M).

\section{Safety}

Throughout the study period, $98.8 \%(82 / 83)$ and $97.6 \%$ (82/84) of patients experienced AEs in the $6 \mathrm{M}$ and $3 \mathrm{M}$ groups, respectively The most common AEs were hot flush, followed by nasopharyngitis, radiation skin injury, injection site induration, injection site pain, white blood cell count decreased, headache and arthralgia, with no significant differences between the 2 groups (Table 3 ). The incidence of a series of injection site reactions (induration, pain, erythema, etc.) was $57.8 \%$ (48/83) and $60.7 \%$ (51/ 84 ) of patients in the $6 \mathrm{M}$ and $3 \mathrm{M}$ groups, respectively.

Most AEs were Grade 1 or 2 in severity. AEs of Grade 3 were reported in $14(16.9 \%)$ and 18 patients $(21.4 \%)$ in the $6 \mathrm{M}$ and $3 \mathrm{M}$ groups, respectively; AEs of Grade 4 were reported in 1 patient $(1.2 \%)$ in the $6 \mathrm{M}$ group. Drug-related 
Table 1 Baseline demographic and disease characteristics of patients (FAS)

\begin{tabular}{|c|c|c|c|}
\hline \multirow[t]{2}{*}{ Variable } & \multicolumn{2}{|l|}{ Treatment group } & \multirow{2}{*}{$\begin{array}{l}\text { Total }(n=167) \\
n(\%)\end{array}$} \\
\hline & $\begin{array}{l}\text { TAP-144-SR }(6 \mathrm{M})(n=83) \\
n(\%)\end{array}$ & $\begin{array}{l}\text { TAP-144-SR }(3 \mathrm{M})(n=84) \\
n(\%)\end{array}$ & \\
\hline \multicolumn{4}{|l|}{ Age (years) } \\
\hline$\leq 39$ & $13(15.7)$ & $12(14.3)$ & $25(15.0)$ \\
\hline $40-44$ & $29(34.9)$ & $30(35.7)$ & $59(35.3)$ \\
\hline$\geq 45$ & $41(49.4)$ & $42(50.0)$ & $83(49.7)$ \\
\hline Mean \pm SD & $44.2 \pm 4.9$ & $44.0 \pm 5.2$ & $44.1 \pm 5.0$ \\
\hline \multicolumn{4}{|l|}{ BMI $\left(\mathrm{kg} / \mathrm{m}^{2}\right)$} \\
\hline Mean \pm SD & $21.5 \pm 3.0$ & $21.5 \pm 2.9$ & $21.5 \pm 3.0$ \\
\hline \multicolumn{4}{|c|}{ Tumor stage (TNM classification) } \\
\hline I & $61(73.5)$ & $61(72.6)$ & $122(73.1)$ \\
\hline IIA & $19(22.9)$ & $21(25.0)$ & $40(24.0)$ \\
\hline IIB & $2(2.4)$ & $2(2.4)$ & $4(2.4)$ \\
\hline IIIA & $1(1.2)$ & $0(0.0)$ & $1(0.6)$ \\
\hline \multicolumn{4}{|c|}{ Tumor size $(\mathrm{cm})$} \\
\hline$\leq 2.0$ & $65(78.3)$ & $66(78.6)$ & $131(78.4)$ \\
\hline$>2.0$ & $18(21.7)$ & $18(21.4)$ & $36(21.6)$ \\
\hline \multicolumn{4}{|c|}{ Number of positive axillary lymph nodes } \\
\hline 0 & $68(81.9)$ & $70(83.3)$ & $138(82.6)$ \\
\hline $1-3$ & $15(18.1)$ & $14(16.7)$ & $29(17.4)$ \\
\hline \multicolumn{4}{|c|}{ ER/PgR expression } \\
\hline $\mathrm{ER}+/ \mathrm{PgR}+$ & $82(98.8)$ & $82(97.6)$ & $164(98.2)$ \\
\hline $\mathrm{ER}+/ \mathrm{PgR}-$ & $1(1.2)$ & $2(2.4)$ & $3(1.8)$ \\
\hline $\mathrm{ER}-/ \mathrm{PgR}+$ & $0(0.0)$ & $0(0.0)$ & $0(0.0)$ \\
\hline \multicolumn{4}{|c|}{ Radiation therapy } \\
\hline Presence & $52(62.7)$ & $59(70.2)$ & $111(66.5)$ \\
\hline Absence & $31(37.3)$ & $25(29.8)$ & $56(33.5)$ \\
\hline \multicolumn{4}{|c|}{ Pre- and postoperative chemotherapy } \\
\hline Presence & $0(0.0)$ & $1(1.2)$ & $1(0.6)$ \\
\hline Absence & $83(100.0)$ & $83(98.8)$ & $166(99.4)$ \\
\hline \multicolumn{4}{|c|}{ Serum estradiol $(\mathrm{pg} / \mathrm{mL})$ at Week 0} \\
\hline Mean \pm SD & $168.0 \pm 163.0$ & $138.2 \pm 125.5$ & $153.0 \pm 145.7$ \\
\hline
\end{tabular}

$F A S$ full analysis set, $B M I$ body mass index, $S D$ standard deviation, $E R$ estrogen receptor, $P g R$ progesterone receptor

Table 2 Suppression rate of serum estradiol to the menopausal levels $(\leq 30 \mathrm{pg} / \mathrm{mL})$ from Week 4 through Week 48 (FAS)

\begin{tabular}{lll}
\hline & TAP-144-SR $(6 \mathrm{M})(n=83)$ & TAP-144-SR $(3 \mathrm{M})(n=84)$ \\
\hline Suppression rate of serum estradiol $[\%(95 \% \mathrm{CI})]$ & $97.6(91.6,99.7)$ & $96.4(89.9,99.3)$ \\
TAP-144-SR $(6 \mathrm{M})-$ TAP-144-SR $(3 \mathrm{M})[\%(95 \% \mathrm{CI})]$ & $1.2(-5.2,7.8)$ & \\
\hline
\end{tabular}

FAS full analysis set, $E_{2}$ estradiol, $C I$ confidence interval

AEs of $\geq$ Grade 3 were anal fistula, blood triglycerides increased, liver function tests abnormal, hyperlipidaemia and interstitial lung disease (1 patient each) in the $6 \mathrm{M}$ group, and gamma-glutamyltransferase increased (3 patients), hypertension (2 patients), weight increased, neutropenia, blood triglycerides increased and interstitial lung disease (1 patient each) in the $3 \mathrm{M}$ group.
Serious AEs (SAEs) were reported in $6(7.2 \%)$ and 7 patients $(8.3 \%)$ in the $6 \mathrm{M}$ and $3 \mathrm{M}$ groups, respectively, and included 3 drug-related SAEs: interstitial lung disease in 1 patient in each group and anal fistula in 1 patient in the $6 \mathrm{M}$ group.

AEs leading to discontinuation of the study drug occurred in $4(4.8 \%)$ and 5 patients $(6.0 \%)$ in the $6 \mathrm{M}$ and 


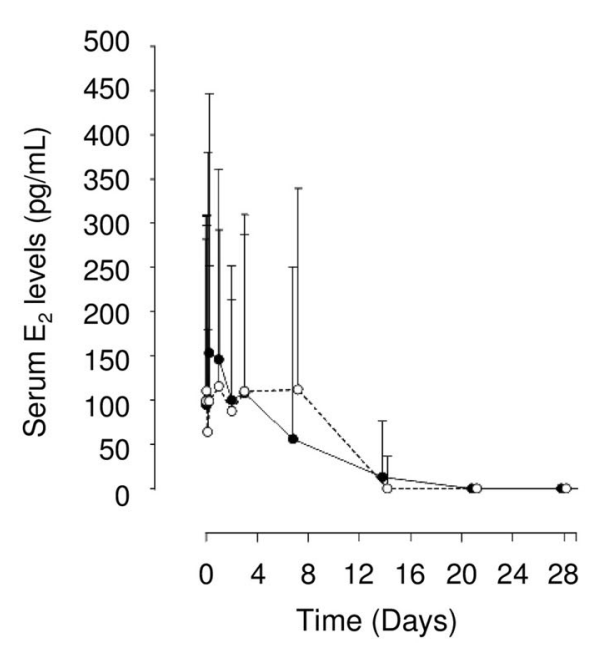

Fig. 2 Time course of serum estradiol concentration from the start of study drug administration through Week 48 (FAS). Data are presented as the median and the 75 th percentile. $E_{2}$ estradiol, FAS full set analysis

Fig. 3 Time course of serum TAP-144 concentration from the start of study drug administration through week 24 (PAS). Data indicate the mean + SD. SD standard deviation, $P A S$

pharmacokinetics analysis set

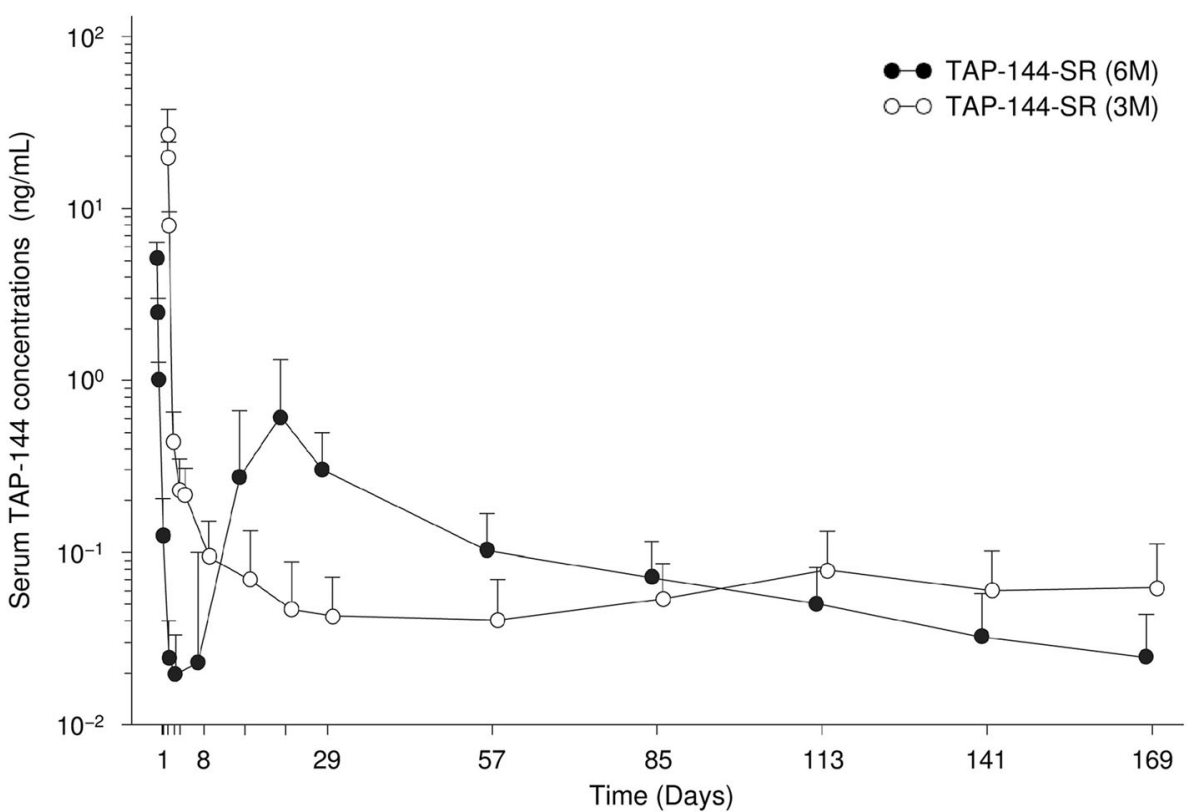

$3 \mathrm{M}$ groups, respectively. Of these, AEs for which a causal relationship could not be ruled out were found in 4 patients (palpitations in 1 patient, joint stiffness, menopausal symptoms, and dry skin in 1, liver function test abnormal in 1 , and interstitial lung disease in 1) in the $6 \mathrm{M}$ group, and in 4 patients (radiation pneumonitis in 1 patient, gammaglutamyltransferase increased in 1, genital hemorrhage in 1 , and interstitial lung disease in 1) in the $3 \mathrm{M}$ group. There were no deaths throughout the study period.

For ECG data analysis, QTcF intervals declined from the baseline values through $6 \mathrm{~h}$ after the study drug administration in both treatment groups, and no transient prolongation of QTcF intervals was detected at around the time of the $C_{\max }$. The mean changes (SD) in $\mathrm{QTcF}$ intervals from baseline were 9.7 (15.36) $\mathrm{ms}$ at Week 4, 9.2 (14.76) $\mathrm{ms}$ at Week 48, and 8.4 (14.73) $\mathrm{ms}$ at Week 96 in the 6M group, and 11.4 (13.83) $\mathrm{ms}$ at Week 4, 10.8 (15.13) $\mathrm{ms}$ at Week 48, and 13.1 (22.03) ms at Week 96 in the 3M group. Prolonged QTcF intervals of approximately $10 \mathrm{~ms}$ were observed from Week 4 through Week 96 in both treatment groups. Prolonged QTcF intervals of $>60 \mathrm{~ms}$ from baseline were reported in 3 patients ( 2 and 1 in the $6 \mathrm{M}$ and $3 \mathrm{M}$ groups, respectively), which were transient and detected only at 1 assessment time point in each patient. At 7 institutions where it was possible to measure ECG at all assessment points, including 3 and $6 \mathrm{~h}$ after the study drug administration, the same ECG for all patients was used, and a total of 54 patients were interpreted at the central reading 
Table 3 Adverse events occurring in $10 \%$ or more of patients in any treatment group (SAS)

\begin{tabular}{lll}
\hline Preferred term $^{\mathrm{a}}$ & $\begin{array}{l}\text { TAP-144-SR }(6 \mathrm{M})(n=83) \\
n(\%)\end{array}$ & $\begin{array}{l}\text { TAP-144-SR }(3 \mathrm{M})(n=84) \\
n(\%)\end{array}$ \\
\hline Patients with any AEs & $82(98.8)$ & $82(97.6)$ \\
Hot flush & $43(51.8)$ & $48(57.1)$ \\
Nasopharyngitis & $47(56.6)$ & $42(50.0)$ \\
Radiation skin injury & $31(37.3)$ & $39(46.4)$ \\
Injection site induration & $36(43.4)$ & $33(39.3)$ \\
Injection site pain & $24(28.9)$ & $26(31.0)$ \\
White blood cell count decreased & $27(32.5)$ & $19(22.6)$ \\
Headache & $21(25.3)$ & $19(22.6)$ \\
Arthralgia & $18(21.7)$ & $20(23.8)$ \\
Malaise & $13(15.7)$ & $13(15.5)$ \\
Injection site erythema & $13(15.7)$ & $8(9.5)$ \\
Musculoskeletal stiffness & $11(13.3)$ & $9(10.7)$ \\
Weight increased & $12(14.5)$ & $8(9.5)$ \\
Back pain & $13(15.7)$ & $6(7.1)$ \\
Insomnia & $10(12.0)$ & $9(10.7)$ \\
Injection site swelling & $12(14.5)$ & $5(6.0)$ \\
Hyperhidrosis & $9(10.8)$ & $7(8.3)$ \\
Nausea & $7(8.4)$ & $9(10.7)$ \\
Constipation & $13(15.7)$ & $2(2.4)$ \\
Dizziness & $6(7.2)$ & $9(10.7)$ \\
Gamma-glutamyltransferase increased & $3(3.6)$ & $12(14.3)$ \\
Rash & $9(10.8)$ & $6(7.1)$ \\
Eczema & $3(3.6)$ & $9(10.7)$ \\
\hline SAS sar & &
\end{tabular}

$S A S$ safety data analysis set, $A E$ adverse event

${ }^{\text {a }}$ MedDRA, version 16.1 center. Prolonged QTcF intervals of approximately $15 \mathrm{~ms}$ were observed from Week 4 through Week 96 in both treatment groups. Similar prolonged QTcF intervals to those observed in the ECG measurements at the institutions were also observed in the measurements at the central reading center.

For BMD of the lumbar spine $\left(\mathrm{L}_{2}-\mathrm{L}_{4}\right)$, the mean change from baseline tended to gradually decline over time in both treatment groups. The mean change rate in BMD from baseline at Week 48 and Week 96 were $-5.1 \%$ (95\% CI -5.8 to -4.5$)$ and $-7.6 \%(95 \% \mathrm{CI}-8.5$ to -6.8$)$ in the $6 \mathrm{M}$ group, and $-4.7 \%(95 \% \mathrm{CI}-5.5$ to -4.0$)$ and $-6.7 \%$ (95\% CI -7.7 to -5.8 ) in the $3 \mathrm{M}$ group. There were no significant differences in the BMD reduction between the treatment groups (Fig. 4).

\section{Discussion}

This is the first report evaluating the efficacy and safety of 6-monthly injections of TAP-144-SR (6M) $22.5 \mathrm{mg}$ in postoperative, premenopausal patients with hormone- receptor positive breast cancer. As for the primary endpoint, TAP-144-SR (6M) was non-inferior to TAP-144-SR (3M) for the effect to suppress the serum $E_{2}$ to the menopausal level from Week 4 through Week 48.

TAP-144-SR (1M) and TAP-144-SR (3M) were effective to suppress the serum $E_{2}$ level to a menopausal level in premenopausal breast cancer patients at the same dose at which suppression of serum testosterone to the castrate level was achieved in prostate cancer patients in clinical studies [14-16]. Therefore, the phase III study was conducted at the injection dose of TAP-144-SR (6M) $22.5 \mathrm{mg}$, which was determined in the phase II study for Japanese treatment-naïve prostate cancer patients [12]. The results showed that the injection dose of TAP-144-SR (6M) $22.5 \mathrm{mg}$, which was successfully used to suppress serum testosterone to the castrate level in prostate cancer patients [17], was also effective in premenopausal breast cancer patients (Fig. 2). In addition, TAP-144-SR (6M) was as effective as TAP-144-SR (3M) to suppress the serum LH and FSH levels from Week 4 through Week 96 in these patients. All patients also achieved amenorrhea from Week 8 , except 1 patient in the $6 \mathrm{M}$ group who experienced 
Fig. 4 Time course of the mean change rates from baseline in bone mineral density of the lumbar spine in patients throughout the 96 week study period (SAS). Data indicate the mean + SD. $S D$ standard deviation, $S A S$ safety analysis set

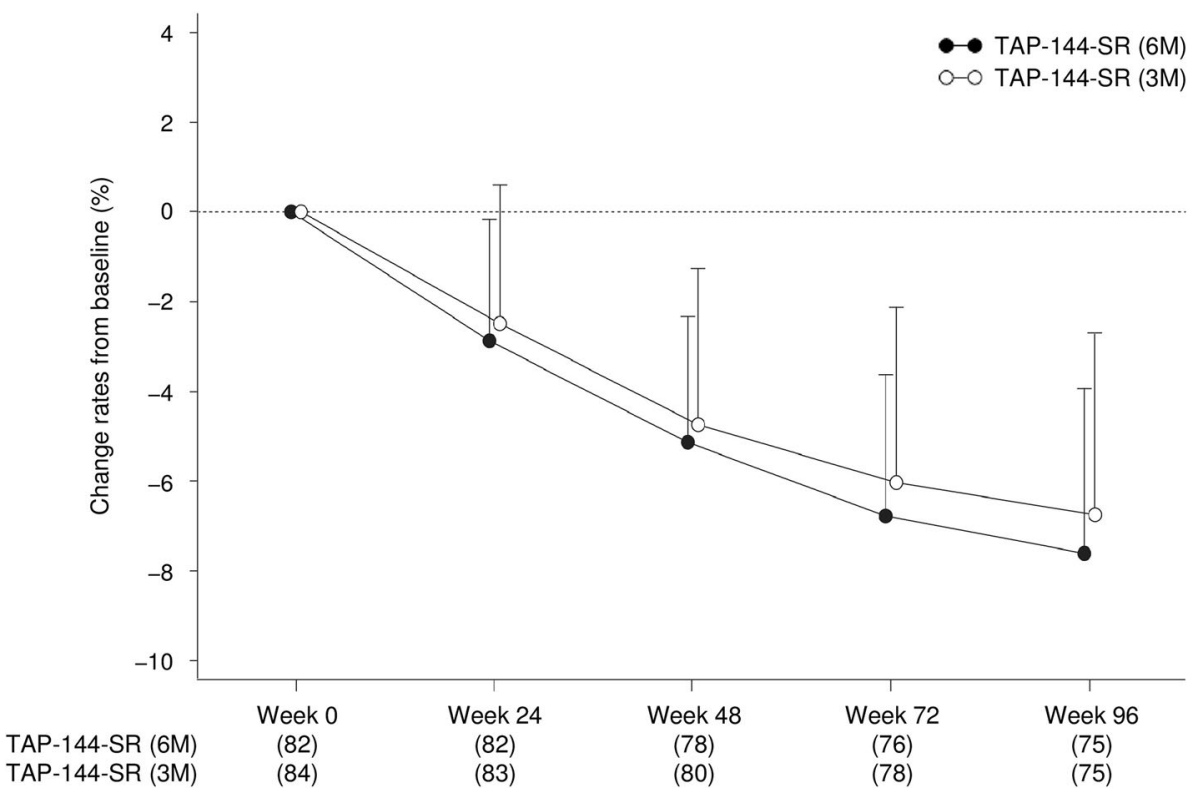

menses at Week 8. It was therefore suggested that ovarian function was substantially suppressed by treatment with TAP-144-SR (6M) $22.5 \mathrm{mg}$ every 24 weeks.

In this study, there were no significant differences in the DFS and DDFS at Week 96 between the groups. There are several reports that more than 3 or 4 years after surgery, the risk of recurrence is higher in ER-positive patients than ERnegative patients $[18,19]$, which may indicate that the risk of recurrence must be reduced by postoperative adjuvant hormone or chemotherapy for a longer period after surgery in ER-positive patients. At the St Gallen International Expert Consensus meetings, both postoperative tamoxifen and tamoxifen plus ovarian function suppression for 5 years were considered acceptable as the standard treatment for premenopausal patients with hormone-receptor positive breast cancer [10]. Furthermore, the results of the Suppression of Ovarian Function Trial (SOFT) showed that 5-year treatment with tamoxifen plus ovarian suppression might provide a benefit for DFS in high-risk patients younger than 35 years who have remained premenopausal after adjuvant chemotherapy, compared to tamoxifen alone [20]. Therefore, long-term administration of an LH-RH agonist is a possibility in high risk premenopausal patients with endocrine-responsive breast cancer.

In the PK analysis, TAP-144-SR (6M) showed a doublepeak of TAP-144, similar to the results in the PK analysis obtained in previous Japanese clinical trials in prostate cancer patients [12], which demonstrated that the clinically sufficient drug concentration to suppress ovarian function was maintained throughout a period of 24 weeks with a single injection (Fig. 3). The serum TAP-144 concentration profile after the initial administration of TAP-144-SR (6M) was similar to that after its second administration.
As for safety, there were no significant differences in the safety profiles and tolerability between the groups, regarding the incidence, type and severity of AEs. The most common drug-related AEs included menopausal symptoms such as hot flush, headache and arthralgia, and injection site reactions (Table 3 ). A series of injection site reactions were reported in about $60 \%$ of patients in each group with no significant between-group differences in the incidence and severity. All of these events were $\leq$ Grade 2 in severity, and no cases led to discontinuation due to injection site reactions.

During the study period, 3 serious drug-related AEs were reported: interstitial lung disease in 1 patient in each treatment group and anal fistula in 1 patient in the $6 \mathrm{M}$ group. Both patients with interstitial lung disease had postoperative radiation and adjuvant endocrine therapy in the same period. It is known that pulmonary fibrosis frequently occurs following radiation therapy, and that tamoxifen may cause the development of lung fibrosis by inducing transforming growth factor- $\beta$. It was also reported that tamoxifen treatment during post-mastectomy radiation in breast cancer patients significantly increased the risk for the development of lung fibrosis along with other prognostic factors like age and menopausal status [21]. Since the pathogenesis of interstitial lung disease is still unclear, further research is necessary to evaluate whether the concomitant implementation of radiation and adjuvant TAP144 plus tamoxifen therapy is associated with interstitial lung disease.

As for the ECG data, there was not a tendency of QTcF interval prolongation around the time of $C_{\max }$, and the mean change in QTcF intervals from Week 4 through Week 96 was approximately $10 \mathrm{~ms}$ in each treatment 
group. It is considered that this QTcF prolongation may not be primarily due to the pharmacological effect of TAP-144, but be secondary to the suppression of ovarian $\mathrm{E}_{2}$ production. A study of the effects of sex hormones on QTcF interval prolongation suggests that estrogen might be a risk factor for drug-induced torsades de pointes. Although $\mathrm{E}_{2}$ may influence clinically relevant QT interval prolongation, the pathogenesis is still not clear [22].

Although BMD tended to gradually decline over time in both treatment groups, the changes in BMD in TAP-144SR (6M) were similar to those of the results of our previous clinical study in TAP-144-SR (3M) (Fig. 4) [23]. It is well recognized that serum $\mathrm{E}_{2}$ level suppression with an LH-RH agonist can cause BMD reduction, which can be prevented or mitigated with the concomitant use of anti-osteoporosis drugs [24].

In this phase III study, the non-inferiority of TAP-144SR (6M) $22.5 \mathrm{mg}$ to TAP-144-SR (3M) at $11.25 \mathrm{mg}$ was confirmed in terms of the suppressive effect on serum $\mathrm{E}_{2}$ to the menopausal level. No clinically significant other differences in efficacy or tolerability were observed between the treatment groups.

TAP-144-SR (6M) allows mitigation of the burden on patients and physicians by reducing the dose frequency of the treatment for premenopausal patients with endocrineresponsive breast cancer. In particular, young premenopausal patients who are busy with work and housework may derive great benefit from TAP-144-SR (6M).

Acknowledgments We are grateful to all the patients, investigators, and clinical research coordinators who participated in this study. The study centers are as follows: Hokkaido Cancer Center, Hokkaido University Hospital, Sapporo Breast Surgical Clinic, Niigata Cancer Center Hospital, Fukushima Medical University Hospital, Gunma Prefectural Cancer Center, Gunma University Hospital, Saitama Cancer Center, Keio University Hospital, Yokohama City University Medical Center, Tokai University Hospital, Nagoya City University Hospital, Kyoto Prefectural Medical University Hospital, Osaka National Hospital, Osaka City University Hospital, Nakanoshima Osaka Breast Clinic, Osaka Medical Center for Cancer and Cardiovascular Diseases, Osaka University Hospital, Kawasaki Medical School Hospital, and Kumamoto University Hospital. Editorial assistance with the preparation of this manuscript was provided by WysiWyg Co., Ltd., and financial support by Takeda Pharmaceutical Company Limited; the authors retained editorial control over the content.

\section{Compliance with ethical standards}

Conflict of interest J. Kurebayashi received advisory/consultation fees from Takeda Pharmaceutical Company Limited and research funding from Takeda Pharmaceutical Company Limited, Eisai Company Limited, Chugai Pharmaceutical Company Limited and AstraZeneca K.K. T. Toyama received a research funding from Takeda Pharmaceutical Co., Ltd., Daiichi Sankyo Healthcare Co., Ltd. and Novartis Pharma K.K. S. Sumino, E. Miyajima and T. Fujimoto are employees of Takeda Pharmaceutical Company Limited. This work was supported by Takeda Pharmaceutical Company Limited.
Open Access This article is distributed under the terms of the Creative Commons Attribution 4.0 International License (http://crea tivecommons.org/licenses/by/4.0/), which permits unrestricted use, distribution, and reproduction in any medium, provided you give appropriate credit to the original author(s) and the source, provide a link to the Creative Commons license, and indicate if changes were made.

\section{References}

1. Goldhirsch A, Glick JH, Gelber RD, Coates AS, Senn HJ. Meeting highlights: International Consensus Panel on the Treatment of Primary Breast Cancer. Seventh International Conference on Adjuvant Therapy of Primary Breast Cancer. J Clin Oncol. 2001;19:3817-27.

2. Jonat W, Kaufmann M, Sauerbrei W, Blamey R, Cuzick J, Namer $\mathrm{M}$, et al. Goserelin versus cyclophosphamide, methotrexate, and fluorouracil as adjuvant therapy in premenopausal patients with node-positive breast cancer: the Zoladex Early Breast Cancer Research Association Study. J Clin Oncol. 2002;20:4628-35.

3. Early Breast Cancer Trialists' Collaborative Group (EBCTCG). Effects of chemotherapy and hormonal therapy for early breast cancer on recurrence and 15-year survival: an overview of the randomised trials. Lancet. 2005;365:1687-717.

4. Schmid P, Untch M, Kossé V, Bondar G, Vassiljev L, Tarutinov $\mathrm{V}$, et al. Leuprorelin acetate every-3-months depot versus cyclophosphamide, methotrexate, and fluorouracil as adjuvant treatment in premenopausal patients with node-positive breast cancer: the TABLE study. J Clin Oncol. 2007;25:2509-15.

5. Klijn JG, Blamey RW, Baccardo F, Tominaga T, Duchateau L, Sylvester R. Combined tamoxifen and luteinizing hormone-releasing hormone (LHRH) agonist versus LHRH agonist alone in premenopausal advanced breast cancer: a meta-analysis of four randomized trials. J Clin Oncol. 2001;19:343-53.

6. Masuda N, Iwata H, Rai Y, Anan K, Takeuchi T, Kohno N, et al. Monthly versus 3-monthly goserelin acetate treatment in premenopausal patients with estrogen receptor-positive early breast cancer. Breast Cancer Res Treat. 2011;126:443-51.

7. The Japanese Breast Cancer Society Practice guideline for breast cancer in 2013 (in Japanese). Tokyo: Kanehara \& Co., Ltd.; 2013.

8. Christinat A, Di Lascio S, Pagani O. Hormonal therapies in young breast cancer patients: when, what and for how long? J Thorac Dis. 2013;5(Suppl 1):S36-46.

9. Regan MM, Pagani O, Fleming GF, Walley BA, Price KN, Rabaglio M, et al. Adjuvant treatment of premenopausal women with endocrine-responsive early breast cancer: design of the TEXT and SOFT trials. Breast. 2013;22:1094-100.

10. Goldhirsch A, Ingle JN, Gelber RD, Coates AS, Thürlimann B, Senn HJ, et al. Thresholds for therapies: highlights of the St Gallen International Expert Consensus on the primary therapy of early breast cancer 2009. Ann Oncol. 2009;20:1319-29.

11. Shiavon G, Smith IE. Status of adjuvant endocrine therapy for breast cancer. Breast Cancer Res. 2014;16:206.

12. Komura E, Fujimoto T, Takabayashi N, Okamoto H, Akaza H. A phase II pharmacological study of leuprolide acetate 6-month depot, TAP-144-SR (6M), in treatment-naïve patients with prostatic cancer who received a single subcutaneous or intramuscular injection. Gan To Kagaku Ryoho. 2014;41:587-93 (in Japanese with English abstract).

13. Newcombe RG. Interval estimation for the difference between independent proportions: comparison of eleven methods. Stat Med. 1998;17:873-90.

14. Aso Y, Kameyama S, Niijima T, Ohmori H, Ohashi T, Murahashi I, et al. Clinical phase III study on TAP-144-SR, an LH-RH 
agonist depot formulation, in patients with prostatic cancer. Hinyokika Kiyo. 1991;37:305-20 (in Japanese with English abstract).

15. Koiso K, Akaza H, Naito S, Usami M, Tsukamoto T, Shimazaki $\mathrm{J}$, et al. Clinical effects of a 3-month formulation LH-RH agonist, TAP-144-SR (3M) in prostate cancer patients. Hinyokika Kiyo. 2002;48:781-95 (in Japanese with English abstract).

16. Boccardo F, Rubagotti A, Amoroso D, Agostara B, Amadori D, Gallo L, et al. Endocrinological and clinical evaluation of two depot formulations of leuprolide acetate in pre- and perimenopausal breast cancer patients. Cancer Chemother Pharmacol. 1999;43:461-6.

17. Suzuki K, Namiki M, Fujimoto T, Takabayashi N, Kudou K, Akaza H. Efficacy and safety of leuprorelin acetate 6-month depot in prostate cancer patients: a phase III, randomized, openlabel, parallel-group, comparative study in Japan. Jpn J Clin Oncol. 2015;45:1168-74. doi:10.1093/jjco/hyv149.

18. Saphner T, Tormey DC, Gray R. Annual hazard rates of recurrence for breast cancer after primary therapy. J Clin Oncol. 1996;14:2738-46.

19. Kiba T, Inamoto T, Nishimura T, Ueno M, Yanagihara K, Teramukai $\mathrm{S}$, et al. The reversal of recurrence hazard rate between ER positive and negative breast cancer patients with axillary lymph node dissection (pathological stage I-III) 3 years after surgery. BMC Cancer. 2008;8:323.
20. Francis PA, Regan MM, Fleming GF, Láng I, Ciruelos E, Bellet $\mathrm{M}$, et al. Adjuvant ovarian suppression in premenopausal breast cancer. N Engl J Med. 2015;372:436-46.

21. Koc M, Polat P, Suma S. Effects of tamoxifen on pulmonary fibrosis after cobalt-60 radiotherapy in breast cancer patients. Radiother Oncol. 2002;64:171-5.

22. de Kam PJ, van Kuijk J, Lillin O, Post T, Thomsen T. The effect of therapeutic and supratherapeutic oral doses of nomegestrol acetate (NOMAC)/17 $\beta$-estradiol (E2) on QTcF intervals in healthy women: results from a randomized, double-blind, placebo- and positive-controlled trial. Clin Drug Investig. 2014;34:413-20.

23. Shiba E, Yamashita $H$, Kurebayashi J, Noguchi S, Iwase $H$, Ohashi Y, et al. A randomized controlled study evaluating safety and efficacy of leuprorelin acetate every-3-months depot for 2 versus 3 or more years with tamoxifen for 5 years as adjuvant treatment in premenopausal patients with endocrine-responsive breast cancer. Breast Cancer. 2015. doi:10.1007/s12282-0150593-z.

24. Gnant M, Mlineritsch B, Luschin-Ebengreuth G, Kainberger F, Kässmann H, Piswanger-Sölkner JC, et al. Adjuvant endocrine therapy plus zoledronic acid in premenopausal women with earlystage breast cancer: 5-year follow-up of the ABCSG-12 bonemineral density substudy. Lancet Oncol. 2008;9:840-9. 